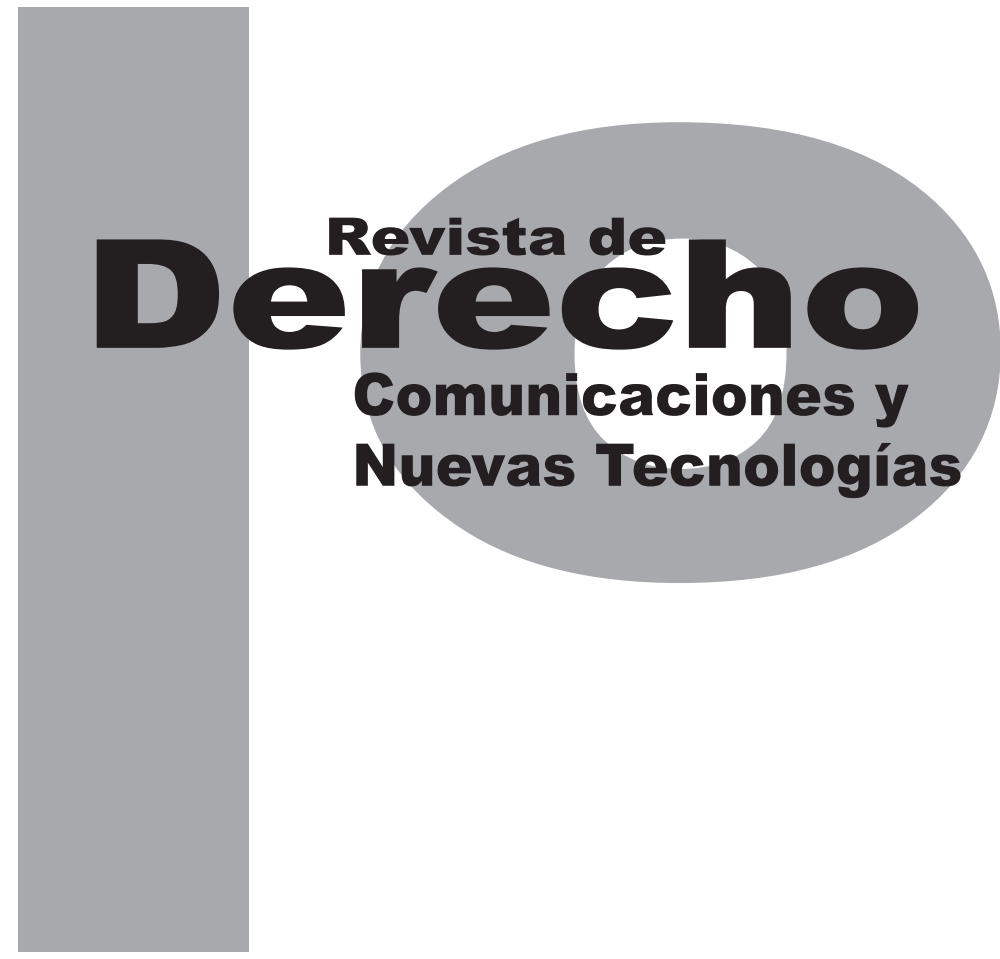

\title{
DEBATES POLÍTICOS: TRATAMIENTO JURÍDICO DE LA ÓRBITA DE LOS SATÉLITES GEOESTACIONARIOS
}

\author{
VALENTINA SANTACOLOMA SÁNCHEZ
}

Universidad de los Andes

Facultad de Derecho

Revista de Derecho, Comunicaciones y Nuevas Tecnologías

No. 10, Julio - Diciembre de 2013. ISSN 1909-7786 


\title{
Debates políticos: tratamiento jurídico de la órbita de los satélites geoestacionarios ${ }^{*}$
}

\author{
Valentina Santacoloma Sánchez ${ }^{* *}$
}

\section{RESUMEN}

El presente trabajo se desarrollará partiendo desde una perspectiva general de la órbita de Ios satélites geoestacionarios, para luego entrar a discutir a grandes rasgos las actuaciones políticas de los Estados y finalmente, centrarnos en las consideraciones colombianas y desarrollos tanto jurisprudenciales como prácticos del tema en cuestión. El enfoque principal de este escrito es analizar los debates políticos que se dieron respecto del tema y las consecuencias que arrojaron las diferentes posiciones adoptadas por los representantes del Estado. En especial haremos un estudio constitucional del tema pasando por varias sentencias de la Corte Consti-

\begin{abstract}
The following article will approach the subject of the geostationary satellite orbit starting from a general perspective; then enter the discussion of political decisions from different countries and finally focus on the Colombian considerations, both jurisprudential and practical developments of the subject matter. The main focus of this paper is to analyze the political debates that took place and the consequences that had the different positions taken by the countries involved. In particular we will study the Constitutional issue though various cases of the Constitutional Court and even the Constitution itself.
\end{abstract}

Cómo citar este artículo: Santacoloma Sánchez, V. (Diciembre, 2013). Debates políticos: tratamiento jurídico de la órbita de los satélites geoestacionarios. Revista de Derecho, Comunicaciones y Nuevas Tecnologías, 10.

** Abogada de la Universidad de los Andes, 2013. Abogada en la Firma LEALANGARITA. Correo: v.santacoloma32@gmail.com , vsantacoloma@lealangarita.com 
tucional hasta revisar el texto mismo de nuestra Constitución.

Palabras CLAVE: Derecho Espacial Internacional, Derecho Constitucional, Espacio Ultraterrestre, Soberanía y Debate político, Órbita Geoestacionaria.
Keywords: International Space Law, Constitutional Law, Outer Space, Sovereignty and Political Debate, Geostationary orbit. 


\section{SUMARIO}

Prefacio - Introducción - I. ÓRBITA DE LOS SATÉLITES GEOSTACIONARIOS - A. Experiencias satelitales en la actividad espacial - B. La órbita de los Satélites Geoestacionarios - 1. Teoría de Arthur C. Clarke 1945 - 2. Punto de vista técnico de la órbita de los satélites geoestacionarios - II. ANÁLISIS JURÍDICO DE LA ÓRBITA DE LOS SATÉLITES GEOSTACIONARIOS - A. Principios rectores del Derecho Espacial: uso pacífico y prohibición de apropiación del Espacio ultraterrestre, la Luna y otros cuerpos celestes - B. Evolución del Problema sobre utilización de la órbita - 1. Tratado de Montreaux 1965 - 2. Tratado de Málaga-Torremolinos 1973 - 3. Trigésima Asamblea General de las Naciones Unidas de1975. Declaración de Bogotá 1976 -4. Conferencia de la UIT sobre Radiofrecuencia 1979 - 5. Tratado de Nairobi 1982 - 5.1 Aplicación práctica del Tratado de Nairobi: algunas cuestiones relativas a las órbitas de los satélites geoestacionarios - III. DEBATES COLOMBIANOS FRENTE A LA PROBLEMÁTICA DE LA ÓRBITA DE LOS SATÉLITES GEOESTACIONARIOS - A. Sentencias de la Corte Constitucional respecto del tema de la órbita de los satélites geoestacionarios - 1. Sentencia C-457 de 1993. Magistrado Ponente Eduardo Cifuentes - 2. Sentencia C-278 de 2004. Magistrado Ponente Manuel José Cepeda Espinosa - B. Tesis del Doctor Alfredo Rey Córdoba respecto de la interpretación del artículo 101 de la Constitución Política - IV. CONCLUSIONES - Bibliografía 
Prefacio

Cuando se introduce el tema del Derecho del Espacio Ultraterrestre tanto lectores como auditorios ponen cara de perplejidad y es que se preguntan ¿pero qué es eso de ultraterrestre? ¿Cómo se regula el espacio si se encuentra tan lejos? ¿Dónde empieza el espacio? Y así, muchos otros interrogantes.

El Derecho Espacial tiene origen en uno de los conflictos internacionales más sonados de la historia: la guerra fría entre la Unión soviética y los Estados Unidos. El único uso del espacio explotado por el hombre hasta entonces era el aéreo, existía la aviación militar pero la altura era mínima, el espacio era completamente diferente. En el espacio aéreo, puesto que existe la densidad de la atmosfera, los objetos navegan a través de ella; a diferencia de lo que sucede en el espacio ultraterrestre, que por falta de atmosfera, los objetos se desplazan. Según Ferrer (1976), los aviones están diseñados para navegar, puesto que el aire es un fluido y el avión debe cortar ese fluido para moverse. Por otro lado, los vehículos espaciales no navegan sino que se desplazan, ya que a pesar de que el vacío posee materia, su ínfima densidad obliga a que las naves espaciales tengan un medio para adquirir velocidad o propulsarse para así lograr desplazarse.

En razón del enfrentamiento bélico entre las dos potencias mundiales del momento (URSS y EE.UU.) se desarrolla una nueva fuerza armamentista tanto por los americanos como por los soviéticos y comienza la conquista del espacio como nuevo territorio de contienda. A pesar de tratarse de un desarrollo de tecnología importante, la comunidad internacional tuvo que intervenir pues no se podía permitir la militarización del espacio conociendo las repercusiones que habían tenido las últimas dos guerras en la humanidad durante la primera mitad del siglo XX. De esta manera, se lleva el tema a las Naciones Unidas para que aborden el problema. Se crea entonces en 1959 la Comisión de utilización del espacio ultraterrestre con fines pacíficos teniendo ésta dos subcomisiones, la primera Subcomisión técnica y científica y la segunda la Subcomisión jurídica. Este es entonces el origen de la normatividad del Derecho Espacial.

El primero de los proyectos presentado por la Subcomisión de asuntos jurídicos fue la Declaración de 1963 que contenía una serie de principios básicos que deben regir las actividades de los Estados en la exploración y utilización del espacio ultraterrestre y los cuerpos celestes. Aunque no tenía la fuerza vinculante de un Tratado internacional "durante el periodo de su aprobación se consideró que constituía la base de un futuro tratado jurídicamente vinculante" (Kopal, 2009, p. 4).

El Derecho del Espacio Ultraterrestre es una rama del Derecho Internacional que se crea a partir de un desarrollo progresivo, cuyos fundamentos son tratados internacionales y resoluciones de la ONU. El hecho de que sea progresivo significa que la mayoría de decisiones se trabajan por consenso y negociaciones entre los países y es de esta manera como se va creando el Corpus Iuris Espacialis. 
El principio filosófico que permea constantemente el Derecho Espacial es la buena fe o, como es llamado en Derecho Internacional, pacta sunt servanda. Aunque los Estados no tengan siempre un fundamento normativo que los obligue a cumplir los mandatos internacionales, este principio de buena fe es lo que los lleva a lograr a diferentes consensos pues en este derecho, lo pactado entre los Estados puede ser de carácter obligatorio. "La confianza en las relaciones internacionales se basa en la fe, en la voluntad de cooperar de otros Estados. La confianza aumentará en la medida en que la conducta de los Estados indique su voluntad de demostrar una actitud no agresiva y de cooperación" (Asamblea de las Naciones Unidas, Examen de directrices para las medidas de fomento de la confianza, 1984 p. 32). Así las cosas, estos pactos de buena fe pueden adoptarse de diversas formas. Pueden concertar la intención de crear obligaciones jurídicas, en cuyo caso representan el Derecho Internacional de tratados entre las partes. Sin embargo, también se pueden concertar mediante compromisos políticos o los llamados consensos. Incluso, dichos compromisos pueden eventualmente convertirse en obligaciones con arreglo al Derecho Internacional, es decir, tratados vinculantes. Es importante resaltar que este tipo de consensos pueden mejorarse paulatinamente a medida que aumenta su aceptación general como norma correcta de conducta: "en consecuencia, la aplicación coherente y uniforme de una medida políticamente obligatoria de fomento de la confianza durante un periodo considerable, junto con la opinio iuirs, puede llevar a la creación de obligación con arreglo al Derecho Internacional consuetudinario" (Asamblea de las Naciones Unidas, 1984, p. 27) Así las cosas, el Derecho Internacional Espacial crece de manera progresiva y dinámica alimentándose tanto de tratados como de consensos para lograr regular el espacio ultraterrestre.

Uno de los más difíciles pero importantes consensos a los que se ha llegado, respecto del espacio ultraterrestre, es sobre su delimitación. En primer lugar, "ultraterrestre" puede entenderse como aquello pertenece al espacio exterior de la Tierra o procede de él, es decir, diferente de la atmosfera que gira con la tierra. Aun así, la atmosfera no es límite entre el Derecho Aéreo y el Derecho Ultraterrestre y siempre ha existido un debate respecto de cuando se acaba el espacio aéreo y cuando comienza el espacio ultraterrestre. ¿La razón? Los países tienen soberanía del espacio aéreo sobre sus territorios, por lo tanto, hasta que se llegue al espacio ultraterrestre se trata de territorio nacional y su imposición como Estado respecto de demás países que requieran transitar por allí.

Los tratados de Derecho Aeronáutico como la Convención de París de 1919 y el Convenio de Chicago de 1944 nunca delimitaron el límite superior del espacio aéreo y cuando comenzaba el espacio ultraterrestre, por eso surgió la necesidad de consensuar al respecto. A través de los tiempos tanto el Subcomité científico como el jurídico han emitido diferentes teorías sobre la delimitación, teorías respecto de las características aerodinámicas de artefactos voladores, la de la gravedad, la teoría de la no delimitación, 
entre otras. Pero siendo este un derecho de consensos, se ha llegado a un punto intermedio, un consenso tácito, puesto que independientemente de la razones técnicas, la mayoría de teorías coinciden en un punto y es que alrededor de los 90 y $110 \mathrm{~km}$ del altitud de la tierra puede decirse que termina el espacio aéreo y comienza el espacio ultraterrestre.

Es de anotar que hasta la actualidad la delimitación y definición del espacio ultraterrestre, tema que ha sido incluido dentro de la agenda del Comité del uso pacífico del espacio ultraterrestre de las Naciones Unidas en adelante COPUS-, no ha podido ser definida con claridad en ningún tratado internacional, es decir, en términos legales estrictos no existe una delimitación exacta, aun así en la práctica existe una aceptación tácita frente a los $100 \mathrm{~km}$ como límite del espacio aéreo y el espacio ultraterrestre.

Así las cosas, nos encontramos frente a un derecho diferente que regula un lugar especial, al cual muy pocos tienen acceso pero que genera todo tipo de controversias y debates en la comunidad internacional. Un lugar sobre el cual todavía queda mucho por descubrir pues presenta desafíos insólitos tanto para la experimentación y funcionamiento de objetos en él, como para su reglamentación y uso por todos y cada uno de los países.

\section{Introducción}

En este trabajo pretendemos hacer un recorrido histórico a través de los debates políticos nacionales e internacionales que han surgido respec- to del tema de la órbita de los satélites geoestacionarios. Un tema polémico que toca aspectos como las diferencias en progresos espaciales entre países desarrollados y en vía de desarrollo, la soberanía de los países y su limitación, la igualdad y equidad en el trato entre países y muchos otros que se irán dilucidando a través del presente artículo. Comenzaremos ubicando al lector en el plano técnico de qué son los satélites geoestacionarios, continuando con una explicación del marco jurídico internacional del debate para luego adentrarnos en la situación colombiana y la discusión constitucional que se ha suscitado al respecto.

Cuando de política se trata, se advierte al lector con la graciosa historia de Stalin y Malenkov: Stalin era un Jefe de Estado de la URSS y se dice que antes de morir dejó para su sucesor dos sobres, uno decía problemas económicos y el otro titulaba problemas políticos. Cuando Stalin murió, Malenkov lo reemplazó en el cargo y comenzó a gobernar. En algún momento de su mandato se le presentó un problema económico y se dirigió al primer sobre que le había dejado Stalin. Allí encontró la respuesta y sacó al país de la crisis económica. Luego de un tiempo Malenkov se encontró en un problema político y se dirigió esperanzado a abrir el segundo sobre, al abrirlo encontró que éste decía "cuando te enfrentes a un problema político escribe 2 sobres"... Sólo queda decir que en materia política la discusión es de nunca acabar. 


\section{I. ÓRBITA DE LOS SATÉLITES GEOSTACIONARIOS}

\section{A. Experiencias satelitales en la actividad espacial}

El Derecho del Espacio Ultraterrestre tiene como función principal la regulación internacional para el uso pacífico del espacio por todos los países y se puede afirmar que el objeto material que estudia esta rama del derecho puede clasificarse en: el espacio ultraterrestre, los cuerpos celestes, las distancias entre los cuerpos celestes y los objetos lanzados al espacio exterior.

En primer lugar tenemos los cuerpos celestes, que son de origen natural perteneciente al espacio dentro o fuera de nuestra galaxia como lo son estrellas, la luna, otros planetas, asteroides, cometas y demás. Todos los cuerpos celestes llevan una fuerza de inercia que es lo que los hace estar en constante movimiento y en diferentes direcciones, avanzan entonces por la cantidad de energía dinámica que los mueve, también conocida como energía oscura (Newton, 1687, p. 145-147). Entre estos cuerpos existen espacios que están en constante cambio debido al movimiento de los cuerpos celestes y las diferentes trayectorias que toma cada uno. Por último tenemos los objetos lanzados al espacio exterior. Entre los objetos espaciales más conocidos tenemos los satélites. El primer satélite fue el SPUTNIK I, lanzado por la Unión Soviética el 4 de octubre de 1957. Fue todo un acontecimiento mundial y a partir de ahí nace la conquista del espacio por parte del hombre.
Antes de ahondar en las experiencias satelitales es importante explicar un elemento esencial para este tema, las órbitas. Como establece Bernardo Ramírez del Valle, la órbita es: "la trayectoria que se describe con relación a un sistema de referencia especificado, el centro de gravedad de un satélite o de otro objeto espacial, por la acción única de fuerzas naturales fundamentalmente por la de gravitación" (Ramírez del Valle, 1985, p. 67. Cursivas mías). Es decir, una órbita existe cuando hay un satélite, cuerpo celeste u objeto espacial que genere ese camino, una órbita no se configura por sí sola debe haber algo que le indique ese recorrido.

Previo al descubrimiento del uso de la órbita de los satélites geoestacionarios, para el tema de las telecomunicaciones se utilizaban las órbitas elípticas que funcionan con un apogeo y un perigeo. El apogeo denomina la distancia máxima que pasa entre el satélite y la tierra, mientras que el perigeo es la distancia mínima que existe entre el satélite y la tierra en su recorrido. Para el funcionamiento de recepción de información de satélites en órbita elíptica se necesitaban varias antenas en la tierra que rastreaban el satélite mientras este se movía y orbitaba, un proceso difícil y costoso porque implicaba seguir al satélite durante todo su recorrido.

Todo eso cambió gracias a Sir Arthur Clarke y su descubrimiento de la órbita de los satélites geoestacionarios, hecho que hizo posible el lanzamiento del primer satélite de órbita geoestacionaria llamado SYNCOM 3, el 19 de agosto de 1964 , ubicado sobre el Ecuador a 180 grados de longitud en el Océano Pacífico. 


\section{B. órbita de los satélites geoestacionarios}

\section{Teoría de Arthur C. Clarke 1945}

En 1945 en su artículo Extraterrestrial Relays, Clarke propone la tesis de la órbita de los satélites geoestacionarios. Esta consistía en que: si un objeto cualquiera se pone a $35875 \mathrm{~km}$. (aprox.) sobre la línea ecuatorial de la tierra, se produce un fenómeno en el cual la fuerza de gravedad de la tierra y la fuerza tangencial que atrae a los objetos hacia fuera, se anulan y el objeto comienza a girar de manera natural alrededor de la tierra a la misma velocidad que ésta. Esto generó toda una revolución en materia espacial pues a diferencia de la órbita elíptica, las antenas ya no tienen que rastrear el satélite sino simplemente acomodarse de cierta manera para que miren hacia el satélite y como éste gira a la misma velocidad de la tierra nunca se pierde de vista.

Agregado a este descubrimiento, Clarke encontró la manera de cubrir toda la tierra con proyección satelital, al colocar tres satélites equidistantes a una altura de $35.875 \mathrm{~km}$. sobre los tres océanos se genera cubrimiento total del globo terráqueo puesto que la proyección de la línea ecuatorial a los $35875 \mathrm{~km}$. es de $360^{\circ}$ generando un círculo a su alrededor.

\section{Punto de vista técnico de la órbita de los satélites geoestacionarios}

Ya habiendo explicado cuál es el fenómeno de la órbita, entraremos a analizar un poco más sobre ésta y sus características.

En primer lugar, se diferencia de otras órbitas, pues un objeto allí colocado gira a la misma velocidad de la tierra. La órbita se encuentra a $35,875 \mathrm{~km}$. de altura sobre la línea ecuatorial y le corresponde un período orbital de $23 \mathrm{~h}$. 56 min. 4 s., el mismo que el de la rotación de nuestro planeta. Para un observador estático en la superficie de la tierra, un satélite ubicado en este lugar con una inclinación de $0^{\circ}$ con respecto al ecuador se percibiría como situado en un punto inmóvil en el cielo. Debido a ello no se necesita un equipo especial de rastreo y las antenas terrestres se apuntan directamente al satélite en forma permanente.

La órbita de los satélites geoestacionarios, consiste en un anillo de $100 \mathrm{~km}$. de ancho, aproximadamente, en sentido Norte-Sur y un espesor de $30 \mathrm{~km}$. que circunda la tierra. Existe porque la fuerza gravitacional de la tierra y la fuerza tangencial del espacio se anulan y por lo tanto en ese punto específico, 35,875 km. de altura aproximadamente, el objeto ahí colocado gira de manera natural alrededor de la tierra a su misma velocidad. Se dice que es una órbita "geo sincrónica" puesto que la velocidad de sus objetos va en sincronía con la velocidad de la trayectoria de la tierra (Bedford \& Fowler, 2000, p. 79). 
La utilidad práctica de esta órbita radica en que es la única que en razón de las características ya enunciadas, permite fijar satélites que giran a la misma velocidad del planeta y no deben rastrearse desde la tierra. Asimismo, por la teoría de la cobertura de proyección satelital de la tierra de Arthur Clarke, con un solo satélite estacionario es suficiente para cubrir una amplia zona de la tierra y se utilizan menos antenas para su observación.

El uso más común para estos satélites es el de las telecomunicaciones, puesto que nunca se pierde su señal ya que siempre está apuntando al mismo punto fijo. Esta sincronía que genera la órbita hace que se reduzcan los costos de rastreo tanto en antenas, como en personal e instalaciones convirtiendo así su uso en una de las modalidades más utilizadas para los satélites de televisión y telecomunicaciones.

Pero aparte de todo esto, ¿Qué es lo que hace que la órbita de los satélites geoestacionarios sea tan importante? Para utilizar la órbita, los satélites deben ubicarse a cinco grados de separación cada uno, o si están ubicados con menos grados de separación deben utilizar frecuencias distintas o, por último, si están muy cerca el uno del otro y usan la misma frecuencia deben radiar partes diferentes del planeta. Esta es la relación de la órbita con el espectro de frecuencias radioeléctricas que conforma el ROE (Recurso Orbita Espectro). De no cumplir con estos lineamientos se puede presentar interferencias entre los satélites afectando así la calidad de sus emisiones.
Lo anterior significa entonces que nos encontramos frente a un recurso natural limitado pues en aquel túnel, de aproximadamente $100 \mathrm{~km}$. de ancho, sólo caben cierto número de satélites con diferentes frecuencias haciendo así que cada vez haya menos espacio en esta órbita tan especial. He aquí donde surge el primer problema político: ¿cómo se reparte este recurso natural especial? ¿Es de todos o de alguien en particular?

\section{ANÁLISIS JURÍDICO DE LA ÓRBITA DE LOS SATÉLITES GEOSTACIONARIOS}

Como se anotó anteriormente, una vez descubierto aquel lugar tan especial, como lo es la órbita de los satélites geoestacionarios, inmediatamente todos los países comenzaron a preguntarse por el trato que debía dársele y el uso correcto de éste. Por fortuna el Derecho Espacial ya había previsto algunos principios y obligaciones comunes que ayudarían a solucionar este debate.

\section{A. Principios rectores del Derecho Espa- cial: uso pacífico y prohibición de apro- piación del Espacio ultraterrestre, la Luna y otros cuerpos celestes}

Desde 1963 la Asamblea General de la Organización de las Naciones Unidas emitió la Declaración de los principios jurídicos que deben regir las actividades de los Estados en la exploración y utilización del espacio ultraterrestre que ya se explicó previamente. En ésta se establecieron las primeras reglas para el uso del espacio, en 
especial, la prohibición de la apropiación y el uso pacífico sin discriminación:

1) La exploración y la utilización del espacio ultraterrestre deberán hacerse en provecho y en interés de toda la humanidad. 2) El espacio ultraterrestre y los cuerpos celestes podrán ser libremente explorados y utilizados por todos los Estados en condiciones de igualdad y en conformidad con el Derecho Internacional. 3) El espacio ultraterrestre y los cuerpos celestes no podrán ser objetos de apropiación nacional mediante reivindicación de soberanía, mediante el uso y la ocupación, ni de ninguna otra manera. (Declaración de los principios jurídicos, 1963, Art. 1-2).

El principio del uso pacífico y sin discriminación del espacio ultraterrestre parte del concepto de res communis ómnium del Espacio, el cual es un bien común de toda la humanidad, explotable y aprovechable por todos los países. Por lo tanto, siendo la órbita de los satélites geoestacionarios parte del espacio ultraterrestre, ésta debía utilizarse pacíficamente y sin discriminación por todos los Estados de acuerdo con los reglamentos de la Unión Internacional de Telecomunicaciones en -en adelante UIT-, los convenios de la misma y del derecho que se vaya creando sobre este tema.

Ahora bien, respecto del concepto de no apropiación éste tiene sus orígenes desde el Derecho Romano y la teoría de dominio y soberanía. En la época romana se creía que existía soberanía sobre el suelo y vuelo, es decir, respecto del territorio y la columna de aire hasta el infinito. Pero con la llegada del Derecho Espacial se ree- valúa dicha teoría por la novedad que presenta la tecnología espacial y cómo se estaba desarrollando en ese tema. Esto es así puesto que, más allá de la atmosfera terrestre al girar la tierra cambia su relación con lo que está fuera de ésta, por lo tanto la columna de aire de la que es dueño un país sólo llega hasta la atmosfera. Así las cosas, el país que reclame soberanía sobre algún sitio del espacio ultraterrestre o cuerpo celeste aduciendo que está en relación a su ubicación terrestre, pierde cualquier credibilidad pues al girar la tierra ya no se encontraría en la misma relación y otro territorio estaría bajo esa misma "línea de dominio".

Así las cosas, a la luz de estos dos principios que luego fueron reiterados en el Tratado de 1967 sobre los Principios que deben Regir las actividades de los Estados en la exploración y utilización del espacio ultraterrestre, incluidos la Luna y otros cuerpos celestes, se creyó resuelto el problema puesto que ese segmento especial era de toda la humanidad y su uso debía ser pacífico y sin discriminación.

Pero como decía el famoso fundador del realismo jurídico Roscoe Pound, hay siempre una diferencia sustancial entre el derecho en los libros y el derecho en la práctica (Pound, 1910, law in books vs. Law in action, p. 2) y aunque los principios dijeran una cosa, en la práctica los Estados argumentaban otra. 


\section{B. Evolución del Problema sobre utiliza- ción y propiedad de la órbita}

\section{Tratado de Montreaux 1965}

En 1965 la UIT aprueba el Convenio de Montreaux. Fue este tratado el último en el que no se habló de la órbita de los satélites geoestacionarios, pero sí se comienza a tratar el tema sobre las frecuencias radioeléctricas.

ARTÍCULO 46. Utilización racional del espectro de frecuencias radioeléctricas:

Los Miembros y Miembros asociados reconocen la conveniencia de limitar el número de las frecuencias y el espacio del espectro utilizados al mínimo indispensable para asegurar de manera satisfactoria el funcionamiento de los servicios necesarios. A tales fines, será conveniente que se apliquen, a la mayor brevedad, los adelantos técnicos más recientes.

ARTíCULO 47 Intercomunicación

1. (300) Las estaciones que aseguren las radiocomunicaciones en el servicio móvil estarán obligadas, dentro de los límites de su empleo normal, al intercambio reciproco de radiocomunicaciones, sin distinción del sistema radioeléctrico que utilicen.

2. Sin embargo, a fin de no entorpecer los progresos científicos, las disposiciones del número 300 no serán obstáculo para el empleo de un sistema radioeléctrico incapaz de comunicar con otros sistemas, siempre que esta incapacidad sea debida a la naturaleza específica de tal sistema y no resultado de dispositivos adoptados con el único objeto de impedir la intercomunicación.
3. No obstante lo dispuesto en el número 300 una estación podrá ser dedicada a un servicio internacional restringido de telecomunicación, determinado por la finalidad de este servicio o por otras circunstancias independientes del sistema empleado. (UIT, 1965 Convenio Internacional de Telecomunicaciones, Montreaux).

\section{Tratado Málaga-Torremolinos 1973}

Para 1973, la UIT hace su primera aproximación concreta al tema de la órbita de los satélites geoestacionarios y en su artículo 33 numeral segundo del Tratado de Málaga-Torremolinos donde se plantea la idea de que la utilización de bandas de frecuencias son recursos naturales limitados así como la órbita en cuestión.

En la utilización de bandas de frecuencias para las radiocomunicaciones espaciales, los Miembros tendrán en cuenta que las frecuencias y la órbita de los satélites geoestacionarios son recursos naturales limitados que deben utilizarse en forma eficaz y económica para permitir el acceso equitativo a esta órbita y a esas frecuencias a los diferentes países o grupos de países, según sus necesidades y los medios técnicos de que dispongan, de conformidad con lo establecido en el Reglamento de Radiocomunicaciones. (UIT, 1973, Convenio Internacional de Telecomunicaciones, MálagaTorremolinos).

Bajo la redacción de este artículo se le estaba dando fuerza a los principios del Derecho del espacio explicados anteriormente (uso pacífico y no apropiación). Aquella res communis que era el espacio ultraterrestre se estaba regulando para que su acceso fuese equitativo y pacífico. 
Con la posterior inclusión del término "equitativo" en 1975, algunos países comenzaron a preguntarse sobre el verdadero acceso que se le estaba dando a este segmento de órbita. El término "igualitario", que no se utiliza en el Tratado Málaga-Torremolinos implica tratar a todas las partes de la misma manera, pero cuando se habla de "equidad" éste incluye la idea de tratar igual a los iguales y de forma diferente a los desiguales, es decir, abogar por el reconocimiento a las diferencias. $Y$ las diferencias que se debían tomar en cuenta, argumentaban algunos, era que respecto de los países que estaban ubicados en la línea ecuatorial estos debían tener un trato diferente a los demás países del mundo. Esto implicaba, probablemente, otorgar ciertas prerrogativas a los países sin tecnología espacial respecto del resto de la comunidad internacional.

Así entonces, el Tratado de Málaga-Torremolinos en su artículo 33, sin proponérselo, despertó uno de los más grandes debates políticos respecto a la órbita de los satélites geoestacionarios. Explosión que luego ocurrió en 1975 en la trigésima Asamblea General de las Naciones Unidas.

\section{Trigésima Asamblea General de las Naciones Unidas de 1975. Declaración de Bogotá 1976}

Previa a la expedición de todos estos Tratados, en 1957 durante la Trigésima Asamblea de la ONU, Colombia plantea la reivindicación de soberanía sobre el segmento que supra yacía su territorio, es decir, entre los grados $70^{\circ}$ y $75^{\circ}$ al oeste de Greenwich aproximadamente. Más tarde, en 1976, durante la Asamblea General reiteró su planteamiento junto al Estado del Ecuador.

De esta manera, en 1976, durante una conferencia de 8 países ecuatoriales que tuvo lugar en Bogotá los Estados de Brasil, Congo, Ecuador, Indonesia, Kenia, Uganda, Zaire y Colombia emitieron una reclamación internacional, conocida como la Declaración de Bogotá, en la cual afirmaron su soberanía nacional sobre el segmento de órbita geoestacionaria que supra yacía a su territorio, por considerarla la tercera dimensión de su soberanía

Los países alegaban que la órbita geoestacionaria no era parte del espacio ultraterrestre, puesto que su existencia dependía exclusivamente a las teorías vinculadas a la ley de la gravitación.

En este sentido, los países signatarios manifestaron que cualquier objeto puesto en dicha órbita requería autorización expresa del Estado afectado, apartándose así de la opinión hasta ese momento imperante según la cual, la órbita geoestacionaria era bien común de la humanidad por estar localizada por fuera del espacio terrestre. (Corte Constitucional, Sentencia C-278 de 2004).

La declaración afirmaba que siendo la órbita de los satélites geoestacionarios un recurso natural limitado, los países que tienen cierta situación especial respecto a ésta tenían el derecho de reclamar soberanía sobre tal segmento. Alegaban que la prohibición de apropiación no regía para el caso y que por falta de definición y delimita- 
ción del espacio ultraterrestre, podían reclamar el segmento de órbita que supra yace a cada país ecuatorial como parte de su territorio. Para avalar semejante manifestación se basaron en la Resolución 2692 (XXV) de las Naciones Unidas (1970), denominada Soberanía permanente sobre los recursos naturales de los países en desarrollo y expansión de la acumulación interna de recursos para el desarrollo económico y además argumentaron que tal como lo señala la Resolución 3281 (XXIV) de la Asamblea General de la ONU (1974), "Todos los estados tienen el libre ejercicio pleno y permanente de la soberanía, incluidas las posesiones, el uso y disposición de todos sus bienes, sus recursos naturales y sus actividades económicas" (Art. 1).

Por último, como si fuera poco, luego de ir contra todas las disposiciones internacionales adoptadas desde el inicio de la exploración del espacio ultraterrestre, la Declaración de Bogotá establece que:

para el emplazamiento de un satélite en la órbita geoestacionaria se requiere de autorización expresa por parte del país sobre cuyo segmento de órbita se ubica el artefacto, además de que la colocación de los mismos no confiere derecho alguno al país que lo realiza. (Declaración de Bogotá, 1976, num. 2).

Como era de esperarse, dicha declaración de los países ecuatoriales causó revuelo en toda la comunidad internacional, que posteriormente tuvo como marco de desarrollo la Conferencia de radiocomunicaciones de 1979 de la UIT en Ginebra.

\section{Conferencia de la UIT sobre radiofrecuencia 1979}

En la conferencia internacional de la UIT dedicada al tema de la radiofrecuencia que se llevó a cabo en 1979, la discusión sobre la declaración de Bogotá seguía aún vigente. Además de que en el transcurso de 1975 hasta 1976 los países signatarios habían incluido aún mas atribuciones a sus Estados por considerarlos especiales respecto de la órbita de los satélites geoestacionarios. Para esta época la declaración aseguraba que:

La órbita geoestacionaria no hace parte del espacio ultraterrestre y los países ecuatoriales ejercen soberanía sobre dicha franja, seguida de otra menos categórica, admitida en parte por algunos instrumentos internacionales (UIT), en donde se reconoce la necesidad de utilizar equitativa y racionalmente la órbita geoestacionaria, sobre la cual el país ejerce una soberanía "no tradicional". (Corte Constitucional, Sentencia C-278 de 2004).

De esta manera, para concluir, los países ecuatoriales: Colombia, El Congo, Ecuador, la República Gabonesa, Kenia, Uganda, La República Democrática Somalí y la República del Zaire emiten una reserva a la Resolución \# 3 de la Conferencia Administrativa Mundial de Radiocomunicaciones de 1979. En dicha reserva establecen que además de lo que afirma dicha Resolución debe entenderse:

La especial situación geográfica de los países ecuatoriales, con respecto a la órbita geoestacionaria, conforme se desprende del desarrollo de los debates habidos en el grupo de traba- 
jo adhoc y en la comisión respectivos. [...] en consecuencia cualesquiera (sic) planificación o reglamentación tendiente a racionalizar la utilización de la órbita geoestacionaria mediante el acceso equitativo de todos los países a la misma, deberá considerarse los planteamientos que al respecto han formulado los países ecuatoriales. (UIT, 1979, Conferencia Administrativa Mundial de Radiocomunicaciones, Art. 79).

Así las cosas, en respuesta a afirmaciones tan enfáticas, se hacen contra reservas a esta declaración por parte del resto de la comunidad Internacional. Acto seguido, todos los países emiten comunicados en los cuales se reservan el derecho de atender sus necesidades de radiocomunicaciones como lo han venido haciendo hasta ahora y de adoptar cuantas medidas consideren necesarias para proteger sus intereses.

En conclusión, el debate político estaba en furor y los oponentes estaban identificados claramente, países ecuatoriales contra los países no ecuatoriales, la comunidad entera se preguntaba: ¿quién tenía la razón y cuál estaría obligado a ceder?

\section{Tratado de Nairobi 1982}

En 1982, en la ciudad de Nairobi capital de Kenia, se toman decisiones importantes respecto del polémico tema de la soberanía de los países ecuatoriales sobre el segmento de órbita de los satélites geoestacionarios.

Desde el tratado de Málaga-Torremolinos de 1973 los países ecuatoriales, entre ellos Colombia, llevaban abogando por un trato diferente a otros países en situación especial con respecto a la órbita; pero como se vio en la Conferencia de 1979, sus propuestas no fueron bien recibidas, por lo tanto debían abordarlo de manera diferente.

En síntesis, decidieron que la tesis de la soberanía era un concepto excluyente para todos los países no ecuatoriales por lo que necesitaban una forma de incluir más países a su causa. Así fue como surgió la idea de abogar no sólo por los países situados en la línea ecuatorial sino por todos los países en vía de desarrollo, que así como ellos, también necesitaban voz frente a la comunidad internacional.

Se dejó de lado tácitamente y de manera estratégica la tesis de la soberanía para adoptar una propuesta menos radical y más comprensiva del tema, una tesis incluyente frente a las demás naciones en situaciones similares, porque así como eran países ecuatoriales también eran países en vía de desarrollo y cuando se representa a un mayor numero hay más posibilidades de ser escuchado. Se comienza entonces un proceso de negociación respecto del tema y dado que la tesis de la soberanía respecto de países ecuatoriales no tuvo acogida, había que buscar otra manera de plantear el problema, es decir proponer un "reparto equitativo de frustraciones".

En resumidas cuentas, fruto de la Conferencia Internacional de Telecomunicaciones reunida en Nairobi en 1982, se convino el Tratado de Nairobi, el cual en su artículo 33 tomó en cuenta las recomendaciones de los países en desarrollo y estableció lo siguiente: 
Utilización racional del espectro de frecuencias radioeléctricas y de la órbita de los satélites geoestacionarios

1. Los Miembros procurarán limitar el número de frecuencias y el espectro utilizado al mínimo indispensable para asegurar el funcionamiento satisfactorio de los servicios necesarios. A tales fines, se esforzarán por aplicar, a la mayor brevedad, los adelantos técnicos más recientes.

2. En la utilización de bandas de frecuencias para las radiocomunicaciones espaciales, los Miembros tendrán en cuenta que las frecuencias y la órbita de los satélites geoestacionarios son recursos naturales limitados que deben utilizarse en forma eficaz y económica, de conformidad con lo establecido en el Reglamento de Radiocomunicaciones, para permitir el acceso equitativo a esta órbita y a esas frecuencias a los diferentes países o grupos de países, teniendo en cuenta las necesidades especiales de los países en desarrollo y la situación geográfica de determinados países. (UIT, 1982, Convenio Internacional de Telecomunicaciones, Art. 33. Subrayado mío).

En conclusión, luego de tantos debates y discusiones entre los países se llegó a un acuerdo en el cual, si bien no aceptaba la proclamación de soberanía de la órbita de los satélites geoestacionarios por parte de ningún país; sí se reconoció la necesidad de dar un trato equitativo a las naciones respecto a la utilización del espacio ultraterrestre y en especial de la órbita geoestacionaria. Es menester de la comunidad reconocer las diferencias entre los Estados y bajo el principio internacional de la cooperación internacional, facilitar el progreso espacial de los países en vía de desarrollo.

A partir de un estudio más reciente, encontramos que desde el 1 de enero del año 2012, entraron en vigor las enmiendas al Convenio adoptadas en la Conferencia de Plenipotenciarios (Guadalajara, 2010). En la última versión del convenio se encuentran consolidadas las Actas Finales de la Conferencia de Plenipotenciarios Adicional (Ginebra, 1992) y las Actas Finales de las Conferencias de Plenipotenciarios (Kioto, 1994; Minneapolis, 1998; Marrakech, 2002; Antalya, 2006; Guadalajara, 2010 y recientemente Dubái 2012). Respecto del tema de la utilización racional del espectro de frecuencias radioeléctricas y de la órbita de los satélites geoestacionarios no ha existido ningún cambio sustancial, salvo que ahora, en vez de encontrarse bajo el artículo 33, se encuentra bajo el artículo 44 y se agregó la necesidad de un uso "racional" de dichas órbitas, modificación realizada por la Conferencia de Plenipotenciarios celebrada en Minneapolis (Estados Unidos de América) en 1998. Aun así, seguimos con el principio de equidad desarrollado en el articulado anterior con la misma redacción y bajo el CAPITULO VII titulado "Disposiciones especiales relativas a las radiocomunicaciones" (UIT, 2011).

Aunque el nuevo articulado no genera ningún cambio importante respecto del principio de equidad, es importante hacer la salvedad de que exige un uso racional de las órbitas asociadas, entre estas la órbita de los satélites geoestacionarios. 
Artículo 44 \#2. En la utilización de bandas de frecuencias para los servicios de radiocomunicaciones, los Estados Miembros tendrán en cuenta que las frecuencias y las órbitas asociadas, incluida la órbita de los satélites geoestacionarios, son recursos naturales limitados que deben utilizarse de forma racional, eficaz y económica, de conformidad con lo establecido en el Reglamento de Radiocomunicaciones, para permitir el acceso equitativo a esas órbitas y a esas frecuencias a los diferentes países o grupos de países, teniendo en cuenta las necesidades especiales de los países en desarrollo y la situación geográfica de determinados países. (UIT, 2011. Subrayado mío).

\subsection{Aplicación práctica del Tratado de Nairo- bi: algunas cuestiones relativas a las órbitas de los satélites geoestacionarios}

Es necesario dejar claro que aunque el Tratado de Nairobi planteó la necesidad de ayudar a las naciones en vía de desarrollo, esto aún no se ha visto en la práctica. Hay que reconocer en este punto el Tratado de 1967 sobre Principios que deben Regir las actividades de los Estados en la exploración y utilización del espacio ultraterrestre, incluidos la Luna y otros cuerpos celestes, en especial su artículo 2 que expresa “El espacio ultraterrestre, incluso la Luna y otros cuerpos celestes, no podrá ser objeto de apropiación nacional por reivindicación de soberanía, uso u ocupación, ni de ninguna otra manera”. Aun así no se puede negar que en el espacio ultraterrestre hay un lugar en que a los $35.875 \mathrm{~km}$. sobre la línea ecuatorial aproximadamente, un objeto puesto allí se comporta de una manera diferente. Por lo tanto, un lugar diferente requiere un régimen jurídico diferente, que respete todavía los principios rectores, en especial el de no apropiación, pero que efectivamente ponga en práctica lo que dicta el artículo 44 del Convenio de la UIT respecto de la equidad para el acceso de la órbita.

Uno de los expertos internacionales en el tema, Alfredo Rey Córdoba plantea lo siguiente: Para lograr una verdadera inclusión de los países en desarrollo en el Derecho Espacial, deben otorgárseles situaciones privilegiadas que los equiparen a otras naciones con más progreso en la exploración y utilización del espacio ultraterrestre. Una de estas situaciones puede ser respecto a la asignación de posiciones orbitales que actualmente se rige bajo el principio "primer llegado, primer servido" que implica que quien primero solicita la posición orbital es quien la recibe. Para encontrar entonces la equidad que predica el Tratado de Nairobi de 1982 puede tomarse en cuenta las calidades y situación especial del país que solicita la posición orbital de la siguiente manera:

- Cuando dos países busquen la misma posición y uno sea desarrollado espacialmente y el otro se encuentre en vía de desarrollo, la UIT debería darle prelación al país menos desarrollado y otorgarle la posición a la nación en vía de desarrollo. Claro está, siempre siguiendo el procedimiento establecido para así garantizar la seguridad jurídica de todas las naciones ${ }^{1}$.

La coordinación de posiciones regulada por la UIT consta de los siguientes pasos: 
- Cuando dos países no desarrollados espacialmente busquen una misma posición, la UIT debe estudiar cuál de los dos ya tuvo acceso previo a la órbita y darle prioridad a aquél que no ha tenido acceso.

- Por último, si la UIT se encuentra frente a dos países en las mismas condiciones ya sea desarrollado espacialmente o no, o con acceso previo, entonces deberá aplicarse el principio "primer llegado, primer servido" para la asignación de la posición orbital.

Esta propuesta tuvo un largo lobby político, como lo tiene cualquier cambio en la legislación internacional. En el año 1996 en la Subcomisión de Asuntos Jurídicos, en su $35^{\circ}$ período de

1. Publicación anticipada: se da aviso a todos los países que se esta solicitando una posición en especial

2. Coordinación: si un país tiene algún problema o comentario al respecto porque podría causarse interferencia se abre una etapa de coordinación entre ambos países.

3. La UIT publica la coordinación de ambos países

4. Se corre traslado para que los demás países se pronuncien respecto de la conciliación. Si no logran hacer compatibles los sistemas de los países en conflicto, la UIT conforma un Tribunal de Arbitramento para dirimir la controversia y asignar el uso de la posición orbital.

5. Generalmente los países coordinan entre sí.

6. Por último, ya sea por medio de un acuerdo en la coordinación o como una consecuencia excepcional el Tribunal de Arbitramento de la UIT asigna el uso de la posición orbital, mas nunca la propiedad porque como indican todas las disposiciones internacionales, la órbita es parte del espacio ultraterrestre y nada de éste es sujeto de apropiación.

7. Junto con la asignación de uso de una posición orbital, también se otorga una frecuencia radioeléctrica para poder manejarlo desde tierra y recibir su señal. (UIT, 2012, Reglamento de radiocomunicaciones, Art.8). sesiones, el delegado de Colombia presenta el proyecto de un régimen jurídico especial titulado Algunos aspectos relativos a la utilización de la órbita de los satélites geoestacionarios (A) AC.105/C.2/L.200 y Corr.1.). Este proyecto no tiene mucha acogida en los países desarrollados, salvo por algún país de la Comunidad Europea que fuera de las votaciones comunica su apoyo a la propuesta. Aun así, dicho Estado explica que no puede votar a favor porque su nación hace parte de la Comunidad Europea y como comunidad tienen una sola posición, que es en contra. Luego de una larga deliberación la Subcomisión decide no respaldar el documento en razón de la cantidad de votos en contra. De todos modos, el delegado de Colombia exhorta a dicha delegación para que le dé una mirada al proyecto y analicen su viabilidad.

Efectivamente, en el $38^{\circ}$ período de sesiones de la Subcomisión de asuntos jurídicos, celebrado en 1999, el Estado perteneciente a la comunidad Europea plantea una vez más la propuesta, pero en esta ocasión lo hace a nombre propio, un país desarrollado que tiene más peso en la comunidad internacional. Finalmente, luego de 4 años de negociaciones dicha Subcomisión adopta el documento y expide el Anexo III titulado "Algunos aspectos relativos a la utilización de la órbita de los satélites geoestacionaria: documento aprobado por la Subcomisión de Asuntos Jurídicos" que se da en el marco de la Reunión de la Subcomisión de Asuntos Jurídicos en su $39^{\circ}$ período de sesiones, celebrado en Viena del 27 de marzo al 6 de abril de 2000. En este, la subcomisión le da reconocimiento a 
Colombia por su ardua labor y además adopta las recomendaciones formuladas en el párrafo 8 del documento.

Actualmente, después de 13 años estamos a la espera de una reglamentación por parte de la UIT, lo cual no significa que estemos por mal camino porque, debemos recordar que el Derecho Espacial es un derecho de desarrollo progresivo y toma tiempo en evolucionar. Por lo tanto, para la asignación de posiciones orbitales de la UIT aún se sigue utilizando el principio de "primer llegado, primer servido".

Ahora bien, habiendo estudiado detalladamente los problemas políticos internacionales que se suscitaron frente a la órbita de los satélites geoestacionarios, queda aún cierto terreno por explorar y éste es el debate interno que vivía CoIombia con la reclamación de su soberanía.

\section{DEBATES COLOMBIANOS FRENTE A LA PROBLEMÁTICA DE LA ÓRBITA DE LOS SATÉLITES GEOESTACIONARIOS}

Es entonces el momento de analizar desde la perspectiva colombiana el tema de la órbita de los satélites geoestacionarios. Paralelo a las discusiones políticas que se estaban dando a nivel Internacional, en el interior del país también se vivía una época de cambio, la cual culminó con la declaración de Colombia como un Estado Social de Derecho y la proclamación de la Constitución Política de 1991, la cual acogió de alguna manera los planteamientos que se desarrollaron en el capitulo anterior. La carta política, en una maniobra que algunos Ilaman atrevida y otros adecuada, en su artículo 101 y 102 reclamó soberanía sobre el segmento de órbita geoestacionaria:

ARTíCULO 101. Los límites de Colombia son los establecidos en los tratados internacionales aprobados por el Congreso, debidamente ratificados por el Presidente de la República, y los definidos por los laudos arbitrales en que sea parte la Nación.

Los límites señalados en la forma prevista por esta Constitución, sólo podrán modificarse en virtud de tratados aprobados por el Congreso, debidamente ratificados por el Presidente de la República.

Forman parte de Colombia, además del territorio continental, el archipiélago de San Andrés, Providencia, Santa Catalina y Malpelo, además de las islas, islotes, cayos, morros y bancos que le pertenecen.

También son parte de Colombia, el subsuelo, el mar territorial, la zona contigua, la plataforma continental, la zona económica exclusiva, el espacio aéreo, el segmento de la órbita geoestacionaria, el espectro electromagnético y el espacio donde actúa, de conformidad con el Derecho Internacional o con las leyes colombianas a falta de normas internacionales.

ARTíCULO 102. El territorio, con los bienes públicos que de él forman parte, pertenecen a la Nación. (República de Colombia, 1991, Constitución política. Subrayado mío).

Prohibido por todos los Tratados e incumpliendo los principios rectores del Derecho Espacial, aun así Colombia se proclamó como señor y 
dueño del segmento de órbita de los satélites geoestacionarios (grados $70^{\circ}$ y $75^{\circ}$ al oeste de Greenwich aproximadamente).

Así pues, habiendo contrariado normas de Derecho Espacial imperantes en todos los países se comienza a debatir en el país sí ¿pueden o no pueden reclamar soberanía?

\section{A. Sentencias de la Corte Constitucional respecto del tema de la órbita de los sa- télites geoestacionarios}

\section{Sentencia C-457 de 1993. Magistrado Ponente Eduardo Cifuentes}

El primero de los intentos por saldar esta confusión lo da el honorable Magistrado de la Corte Constitucional Eduardo Cifuentes Muñoz en su Sentencia C-457 de 1993. En esta ocasión la Corte debía efectuar un control previo, automático e integral de constitucionalidad respecto de los proyectos de tratados internacionales que celebre el Presidente de la República y sobre las leyes aprobatorias de los mismos, en especial la Ley 252 de 1995. En su análisis del caso el Magistrado Cifuentes le pide un concepto al experto en Derecho Espacial Alfredo Rey Córdoba para que lo ilustre sobre el tema.

En el concepto rendido se explica porqué no debe reclamarse soberanía sobre algo no apropiable en el Derecho Espacial. Asimismo, además de violar uno de los pilares de este derecho; al reclamar soberanía sobre un segmento de órbita del espacio ultraterrestre, aduciendo relación directa con su territorio, se está cayen- do en un error monumental pues se está obviando el principio de rotación de la tierra sobre su misma órbita y alrededor del sol. Como se explicó anteriormente, si se reclama dominio sobre un segmento del espacio ultraterrestre o sobre un cuerpo celeste, con base en que éste radia sobre su territorio, en menos de un segundo esa tesis se caería puesto que a medida que la tierra rota sobre su propio eje esa relación con el segmento o cuerpo celeste desaparece y la radiación se genera sobre un territorio diferente. Por último, el Doctor Rey Córdoba enfatiza en la necesidad de un régimen jurídico que:

consagre unos derechos preferenciales para los países en desarrollo, partiendo de los principios de los artículos mencionados de los distintos actos constitutivos, en los que se consagra el acceso equitativo al uso de la órbita, teniendo en cuenta las necesidades especiales de los países en desarrollo y la situación geográfica de determinados países. El experto concluye que la tesis de la soberanía es excluyente e impráctica, mientras que la teoría que aboga por los derechos preferenciales es atractiva para los países que aún no han accedido a posiciones en la órbita geoestacionaria. (Corte Constitucional, Sentencia C -457 de 1993).

De esta manera, la tesis del Doctor Rey es acogida por la Corte y se concreta en la sentencia ya citada. Aunque la Corte Constitucional se declara inhibida sobre la revisión de constitucionalidad de la Ley 28 de 1992 y de los Instrumentos públicos que por ella se aprueban, el tema de la órbita de los satélites geoestacionarios se deja planteado para luego ser retomado por el 
Magistrado Manuel José Cepeda Espinosa unos años después.

\section{Sentencia C-278 de 2004. Magistrado Ponente Marco Gerardo Monroy Cabra y Manuel José Cepeda Espinosa}

En el 2004 el debate vuelve a surgir, pero está vez bajo la reforma de la Organización Internacional de Satélites de Telecomunicaciones -en adelante INTELSAT- que luego se convertirá en la Organización Internacional de Telecomunicaciones -en adelante ITSO-. En esta sentencia se analiza el acuerdo que determina la renovación de INTELSAT y dispone las medidas necesarias para su privatización, así como para la entrega de la supervisión de la sociedad INTELSAT Ltda. a la ITSO. La mayor parte del fallo analiza si el acuerdo se ajusta a la Constitución y en su mayoría supera el examen de constitucionalidad, aun así, en el acuerdo se toca el tema de la órbita geoestacionaria y siendo ésta "propiedad reclamada" por Colombia es obligación de la Corte pronunciarse al respecto. La sentencia hace una recopilación de todos los debates internacionales que han surgido al respecto y la evolución de estos hasta el momento, todo para llegar al análisis de artículo 101 y 102 de la Constitución política. A través de un exhaustivo estudio del tema la Corte hace hincapié en el principio de no apropiación que rige el uso del espacio ultraterrestre:

De hecho, la doctrina internacional admite que como, desde el lanzamiento de los primeros cohetes al espacio exterior por parte de los EE.UU. y la entonces Unión Soviética, ningún país presentó formalmente reclamación alguna en torno a la posible indebida utilización del espacio que pudiera considerarse como nacional, se instauró en el ámbito internacional una norma consuetudinaria -instant custom- que ha venido a hacer carrera en los instrumentos internacionales posteriores. La norma internacional, así conformada, constituye fundamento del principio del espacio ultraterrestre como res communis omnium (SIC), o bien de toda la humanidad. (Corte Constitucional, Sentencia C-278 de 2004).

Por consiguiente, luego de desglosar todas las actuaciones de la comunidad internacional, así como las diferentes posiciones que ha tenido Colombia en el debate, la sentencia esgrime, en mi opinión, un argumento un poco escueto para matizar la soberanía que reclama Colombia sobre el segmento de la órbita de los satélites geoestacionarios.

Es entonces necesario criticar la labor de la Corte en esta materia, pues tenían razones y fundamentos suficientes para definir el tema y actuar según las directrices internacionales y aceptar finalmente que Colombia no puede ser dueño de un bien del que no está permitido apropiarse (Tratado, 1967). Aun así, no sabemos si es por miedo, falta de convicción o sólo que optaron el camino fácil de no dar la razón a nadie y en consecuencia decidieron lo siguiente:

No obstante, de la lectura detenida del artículo 101 se tiene que la órbita geoestacionaria es parte del territorio colombiano, "de conformidad con el Derecho Internacional o con las leyes colombianas a falta de normas internacionales", de lo cual se deduce que la norma- 
tividad del Derecho Internacional no es irrelevante para verificar el ejercicio de la soberanía nacional sobre la misma. Es más, el ejercicio de la soberanía sobre dicho segmento de la órbita debe ejercerse de acuerdo con el Derecho Internacional, según las voces de este artículo de la Carta.

(...) No obstante, visto el desarrollo de los argumentos expuestos, esta decisión debe respetar el contenido de los artículos 101 y 102 de la Carta, que declaran el segmento de órbita geoestacionaria como parte del territorio nacional. En el mismo sentido, dicho reclamo debe ser consecuente con la posición que vincula la definición de la órbita geoestacionaria con las normas internacionales y con aquella que reconoce la inmadurez normativa de este campo del derecho. Esto exige que la declaratoria de exequibilidad del acuerdo respete la pertenencia de la órbita al territorio nacional y permita que Colombia siga intentando obtener ante la comunidad internacional el reconocimiento de los derechos que por su específica posición considere tener sobre el segmento de la órbita. (Corte Constitucional, Sentencia C-278 de 2004. Subrayado mío).

Así las cosas, la Corte Constitucional condiciona la soberanía de la órbita referida de la siguiente manera: 1) De conformidad con el Derecho Internacional, es decir, siempre teniendo en cuenta las normativas internacionales frente al tema y 2) [de conformidad] con las leyes colombianas a falta de normas internacionales; en este caso priman las normas internacionales frente las nacionales y como es comúnmente sabido sí existen normas que regulen el tema (Tratado, 1967).
Por lo tanto, habría que llegar a la conclusión, después de interpretar la sentencia ya citada, que Colombia no puede reclamar soberanía sobre el segmento de la órbita de los satélites geoestacionarios ya que los tratados internacionales respecto del espacio ultraterrestre prohíben la apropiación de cualquier bien, objeto $u$ cuerpo celeste del espacio ultraterrestre.

No obstante a pesar de haber concluido el tema con una pobre decisión al respecto, que exige una exhaustiva hermenéutica por parte del lector de normativas internacionales, la sentencia hace una buena labor en retomar la propuesta explicada anteriormente, de lograr un trato equitativo para los países en vía de desarrollo respecto de la asignación de posiciones orbitales por parte de la UIT. Para profundizar en el análisis, a continuación se retoma la posición del experto en el tema Alfredo Rey Córdoba, desglosando el artículo 101 de la Constitución y explicando qué significa aquella soberanía condicionada.

\section{B. Tesis del Doctor Alfredo Rey Córdoba respecto de la interpretación del artículo 101 de la Constitución Política}

Para dejar claro de una vez por todas por qué Colombia no puede reclamar soberanía del segmento de la órbita de los satélites geoestacionarios (grados $70^{\circ}$ y $75^{\circ}$ al oeste de Greenwich aproximadamente) y desglosar el condicionamiento que hace la Corte Constitucional del artículo 101 se explicarán los siguientes temas: i.) Convención de Viena, Declaración de 1963 y Tratado de 1967 y ii.) Costumbre Internacional. 
En primer lugar siendo el Derecho Espacial un derecho autónomo pero que surgió del Derecho Internacional, éste tiene como fundamento para su interpretación la Convención de Viena de 1969. Esta convención es a la que se acude para dilucidar cualquier duda respecto de la interpretación de los tratados, asimismo, sirve de guía a falta de una norma en particular al respecto. De esta manera, como lo proclama la Convención de Viena, los tratados deben entenderse como el conjunto de normas que determinan las directrices para los Estados de la comunidad internacional. Por lo tanto, a pesar de que Colombia no haya ratificado el Tratado de 1967 sobre los principios que deben regir las actividades de los Estados en la exploración y utilización del espacio ultraterrestre, incluso la Luna y otros cuerpos celestes, sí firmo La Declaración de los principios jurídicos que deben regir las actividades de los Estados en la exploración y utilización del espacio ultraterrestre de 1963. En dicha declaración en el numeral $3^{\text {ro, }}$ se establece que: "El espacio ultraterrestre y los cuerpos celestes no podrán ser objeto de apropiación nacional mediante reivindicación de soberanía, mediante el uso y la ocupación, ni de ninguna otra manera." (Declaración de los principios jurídicos, 1963. Subrayado mío).

De esta manera, al hacer un análisis de todos los tratados del Corpus luris Espaciale, encontramos que aunque la Declaración de 1963 es una Resolución no vinculante existen otras razones por las cuales el principio de no apropiación sí es aplicable. Como se dijo anteriormente, el Tratado que tiene fuerza de ley respecto del tema es el Tratado de 1967 y Colombia hasta el momento no lo ha ratificado, pero volviendo a la Convención de Viena respecto de la interpretación de los tratados, ésta en su artículo 38 dispone lo siguiente:

Artículo 38. Normas de un tratado que lleguen a ser obligatorias para terceros Estados en virtud de una costumbre internacional. Lo dispuesto en los artículos 34 a 37 no impedirá que una norma enunciada en un tratado llegue a ser obligatoria para un tercer Estado como norma consuetudinaria de Derecho Internacional reconocida como tal. (Convención de Viena, 1969).

Así las cosas, aunque no se haya ratificado el Tratado de 1967 en el cual se establece como principios de Derecho Espacial entre otros, la no apropiación, ésta premisa es reconocida internacionalmente por todos los países como ya se ha demostrado a lo largo de este artículo. El principio de no apropiación es entonces fruto del desarrollo de la Costumbre Internacional de todos los países, surgiendo así el principio del espacio ultraterrestre como res communis ómnium, el cual significa que el espacio ultraterrestre es una cosa de toda la humanidad y no puede ser adueñada por ningún Estado. Siendo entonces Costumbre internacional la tercera fuente de Derecho Espacial, se reafirma una vez más que Colombia no puede ser dueño del segmento de órbita de los satélites geoestacionarios porque la órbita está en el espacio ultraterrestre y el Tratado de 1967 establece que el espacio no es apropiable. Por lo tanto, puesto que Colombia sí ratificó la Convención de Viena de 1969 se obliga a cumplir todo su articulado y en este caso a reconocer como norma obliga- 
toria una regla consuetudinaria aceptada por la comunidad internacional.

Respecto del artículo 38 de la Convención, existe una manera de no cumplir este mandato y es si ha existido una oposición persistente por parte del Estado frente al tema. En el caso en cuestión, Colombia no podría argumentar que la norma consuetudinaria no le es aplicable porque no ha existido por su parte una oposición persistente y continua, prueba de esto son los siguientes escenarios:

Las declaraciones de soberanía han sido constantes y reiteradas durante los años que han transcurrido desde que comenzó el debate;

Aunque han existido algunas manifestaciones al respecto, la mayoría de éstas sólo han sido en escenarios de dialogo abierto como reuniones de las Naciones Unidas a partir de declaraciones y reservas. Pero en la práctica no se ha proferido ni una sola nota de protesta frente a los Estados que tienen satélites posicionados en el segmento de órbita que Colombia reclama como propio.

En Derecho de petición presentado el 21 de febrero de 2013 a la Agencia Nacional del Espectro ANE, se solicita un informe de la cantidad de satélites geoestacionarios que existen actualmente operativos en el pretendido segmento que reclama Colombia y a qué países pertenecen éstos (entre los grados $70^{\circ}$ y $75^{\circ}$ al oeste de Greenwich aproximadamente). En la contestación se relacionan 147 satélites que se encuentran en proceso o con publicación anti- cipada y se conoce, aproximadamente, de 9 satélites operativos. Por lo tanto, es evidente que no existe una oposición persistente por parte de Colombia en este caso, muestra de lo anterior es que en el pretendido territorio nacional se encuentran 9 objetos espaciales de diferentes países y frente a ninguno se ha presentado un reproche formal.

De igual manera, en Derecho de petición presentado el 21 de febrero de 2013 al Ministerio de Tecnologías de la Información y las Comunicaciones, se le pregunta al Ministerio respecto de la actitud de Colombia frente a los satélites ubicados en el segmento de órbita geoestacionaria reclamada por Colombia. En su respuesta la Jefe de la oficina internacional se limita a citar la Constitución, la Ley 1900 de 1990 y la Ley 1341 de 2009. La primera ley reforma las normas y estatutos que regulan las actividades y servicios de telecomunicaciones y afines. Es tan poco el conocimiento respecto del tema, que el Ministerio incluso cita una ley que fue derogada por el artículo 32 de la Ley 1150 de 2007. Respecto de la segunda ley referida, ésta define principios y conceptos sobre la sociedad de la información y la organización de las Tecnologías de la Información y las Comunicaciones -TIC-, además de crear la Agencia Nacional de Espectro. Dentro de todo su articulado no hay ni una referencia a la órbita de los satélites geoestacionarios. Así las cosas, ni el mismo Ministerio sabe cuál ha sido la posición de Colombia frente al tema, ¿la razón? No se ha pronunciado nunca al respecto, se limita a dar declaraciones ambiguas pero cuando se confronta al tema específico no ha existido ningún tipo de reproche a los 
demás Estados que utilizan su pretendido segmento de la órbita.

En conclusión, podemos argumentar firmemente que a Colombia le es aplicable el artículo 38 de la Convención de Viena de 1969 respecto de la interpretación de los tratados y que el principio de no apropiación le es exigible puesto que se trata de una norma consuetudinaria respecto de la cual no ha existido por su parte una oposición realmente persistente.

\section{CONCLUSIONES}

Para concluir, el debate político que ha surgido desde el descubrimiento de la órbita de los satélites geoestacionarios, ha sido complejo y siempre en evolución. Han existido posiciones radicales que luego se matizan, incluso reformulaciones de tesis únicamente como maniobras políticas para lograr bloques más fuertes; en fin, ha sido toda una contienda de nunca acabar, en la cual Colombia ha jugado un papel preponderante y ha demostrado su liderazgo en el tema del desarrollo espacial. Queda por ver la evolución de sus múltiples propuestas y esperar por la adopción de sus mayores aportes a favor de los países en vía de desarrollo, en especial la propuesta contenida en el documento "Algunos aspectos relativos a la utilización de la órbita de los satélites geoestacionaria: documento aprobado por la Subcomisión de Asuntos Jurídicos". La Resolución 80 de la UIT marcará un gran cambio en el tema del Derecho Espacial colombiano y esperemos que luego de 40 años desde el Tratado de Málaga-Torremolinos, veamos materializado ese acceso equitativo al espacio ultraterrestre que se ha venido promoviendo.

En relación con nuestra Corte Constitucional, ya se ha criticado bastante, pero no debe darse por culminados todos sus intentos de esclarecer el tema de la soberanía Colombiana sobre el segmento de la órbita de los satélites geoestacionarios en cuestión. A fin de cuentas estamos en un Estado Social de Derecho que vela por la protección de su Carta política y que cuenta con una Corte Constitucional que es la guardiana de la misma. Tal vez en algún momento con una Corte más decidida se aventure a saldar el tema del segmento de la órbita de los satélites geoestacionarios y no sea necesario leer entre líneas para finalmente reconocer que dentro del territorio colombiano no puede estar un segmento de la órbita de los satélites geoestacionarios.

Así las cosas, el debate político Colombiano e Internacional respecto de la órbita de los satélites geoestacionarios parece no haber terminado aún, pero al fin de cuentas ¿cuándo termina en realidad una discusión política? Podemos concluir entonces que no se puede reclamar autoridad sobre ningún lugar del espacio ultraterrestre; aún así debe reconocerse que Colombia despertó el tema de la órbita de los satélites geoestacionarios desde su proclamación de soberanía. De igual manera no puede desconocerse que a $35.875 \mathrm{~km}$. sobre la línea ecuatorial, aproximadamente, existe un lugar en el que un objeto allí colocado se comporta con respecto a la tierra de una manera diferente, ergo, ese lugar necesita un régimen jurídico distinto para el cual presentamos la siguiente propuesta: 
A manera de proposición para la UIT en su comité de reglamentación, este escrito quisiera presentar un ejercicio de clase llevado a cabo en la materia de Derecho del Espacio Ultraterrestre, dictado por el profesor Alfredo Rey Córdoba en la Universidad de los Andes (periodo 2013-10). Este ejercicio exhortó a los estudiantes a desarrollar el régimen jurídico especial propuesto para la órbita de los satélites geoestacionarios y formular las posibles hipótesis en cuanto al acceso de las posiciones orbitales teniendo en cuenta el principio de equidad y los países en desarrollo.

La estudiante Sylvana Peña desarrollo la siguiente propuesta:

En el documento aprobado por la Subcomisión de Asuntos Jurídicos en su 39 periodo de sesiones (A/AC.105/738, anexo III) "Algunos aspectos relativos a la utilización de la órbita geoestacionaria" se establecen los procedimientos a seguir que deberían regir en el régimen jurídico especial para la órbita geoestacionaria teniendo en cuenta el fenómeno natural que se presenta a los 35.875 $\mathrm{km}$. sobre la línea ecuatorial de la tierra. En el numeral 8 se presentan entonces las tres hipótesis respecto de la accesión _(SIC) a las posiciones orbitales cuando sea necesaria la coordinación, teniendo en cuenta el principio de equidad y los países en desarroIlo establecidos en la Convención de Nairobi de 1982 y en el artículo 44 de la Constitución de la UIT.

La primera y segunda de estas hipótesis establece que "en caso de solicitudes equiparables para acceder al recurso órbita/espec- tro por parte de un país que ya tenga acceso a dicho recurso y un país en desarrollo $u$ otro país que trate de acceder a él, el país que ya tenga ese acceso debe adoptar todas las medidas viables para permitir que el país en desarrollo o el otro país tenga acceso equitativo al recurso órbita/espectro solicitado" (Algunos aspectos relativos a la utilización de la órbita geoestacionaria, 2000). Es decir que cuando se presente el caso de coordinación de un país que ya accedió con un país que no ha accedido, o de un país desarrollado con un país en vía de desarrollo debería evitarse el proceso de coordinación y dar registro a la posición orbital al país que no ha accedido o al país en vía de desarrollo. Por otro lado, se establece el caso que rige actualmente en el sistema de registro del principio de "quien llega primero, tiene prioridad", cuando se presentan casos de coordinación entre países desarrollados. No obstante se pueden encontrar otros casos en donde un país solicite una posición orbital y deba entrar a coordinar con otros países:

1. País que ya accedió - Primacía a país que no ha accedido

2. País que ya accedió - País en vía de desarrollo: Debería tener prelación el país en vía de desarrollo sin necesidad de coordinación. Sin embargo, si este país en vía de desarrollo ya accedió a una posición orbital se debería entrar a coordinar bajo el principio de "primer llegado, primer servido".

3. País que ya accedió - País desarrollado: Principio de "primer llegado, primer servido".

4.País que ya accedió - País que ya accedió: Principio de "primer llegado, primer servido". 
5. País que no ha accedido - País en vía de desarrollo: Si el país en vía de desarrollo ya accedió se debe dar prelación al país que no ha accedido. Si el país en vía de desarrollo no ha accedido se debe seguir el procedimiento de coordinación bajo el principio de "quien llega primero, tiene prioridad".

6. País que no ha accedido - País desarrollado: Debería tener prelación el país que no ha accedido sin necesidad de coordinación.

7. País que no ha accedido - País que no ha accedido: Debería darse el proceso de coordinación bajo el principio de "quien llega primero, tiene prioridad". No obstante, si alguno de los países es un país en vía de desarrollo debería dársele prioridad a este sin necesidad de coordinación.

8. País en vía de desarrollo - País en vía de desarrollo: Si alguno de los países no ha accedido se le debe dar prioridad a este sin coordinación. De lo contrario se debe entrar a coordinar bajo el principio de "quien Ilega primero, tiene prioridad".

9. País en vía de desarrollo - país desarrollado: Primacía país en vía de desarrollo.

10. País desarrollado - país desarrollado: Principio "primer llegado, primer servido". (2013, “¿Cuáles son las hipótesis posibles en cuanto a la accesión a las posiciones orbitales teniendo en cuenta el principio de equidad y los países en desarrollo? p. 1- 2. Cursivas mías).

Así las cosas, con el documento anterior se quiere exhortar a la UIT para que reglamente el tema y explore las posibilidades que se pueden presentar respecto del acceso a la órbita de los satélites geoestacionarios y cómo se debe tener en cuenta a los países en vía de desarrollo.

\section{Bibliografía}

Asamblea General de las Naciones Unidas. (1963). Declaración de los principios legales que deben regir las actividades de los Estados en la exploración y uso del espacio ultraterrestre. Aprobada por la Resolución 1962 (XVII), de 13 de diciembre de 1963. Recuperado de http://www.oosa.unvienna.org/pdf/ publications/STSPACE11S.pdf

---. (1969). Convención de Viena sobre el Derecho de Tratados. U.N. Doc A/CONF.39/27. Recuperado de http://www.derechos.org/nizkor/ley/viena.html

---. (1967). Tratado sobre los principios que deben regir las actividades de los Estados en la exploración y utilización del espacio ultraterrestre, incluso la Luna y otros cuerpos celestes. Aprobado por la Resolución 2222 (XXI), de 19 de diciembre de 1966. Recuperado de http://www.oosa.unvienna.org/pdf/publications/STSPACE11S.pdf

---. (1970). Soberanía permanente sobre los recursos naturales de los países en desarrollo y expansión de la acumulación interna de recursos para el desarrollo económico. Resolución 2692 (XXV) del 11 de diciembre de 1970. Recuperado de http://daccess-dds-ny.un.org/ doc/RESOLUTION/GEN/NRO/353/53/IMG/ NR035353.pdf?OpenElement 
--. (1984). Examen de directrices para las medidas de fomento de la confianza. Examen de aplicación del documento de clausura del duodécimo periodo extraordinario de sesiones de la asamblea general. Aprobado por la Resolución 39/63 de 12 de diciembre de 1984. Recuperado de http:// daccess-dds-ny.un.org/doc/RESOLUTION/ GEN/NRO/467/90/IMG/NR046790. pdf?OpenElement

--. (2000). Algunos aspectos relativos a la utilización de la órbita de los satélites geoestacionaria: documento aprobado por la Subcomisión de Asuntos Jurídicos. Informe de la Subcomisión de Asuntos Jurídicos sobre su $39^{\circ}$ período de sesiones, celebrado en Viena del 27 de marzo al 6 de abril de 2000, 23-24. Recuperado de http://www.unoosa.org/pdf/ reports/ac105/LGLR00S.pdf

Bedford, A., \& Fowler, W. (2000). Dinámica: mecánica para ingeniería. En: Mecánica de Órbitas. (Vol. 1, pp. 50-85). Upper Saddle River, New Jersey, EE.UU.: Pearson Education.

Clarke, A. (1945). Extraterrestrial Relays. Can Rocket Stations Give World-wide Radio Coverage? Recuperado de http://lakdiva.org/ clarke/1945ww/1945ww_oct_305-308. html

Congreso de la República de Colombia. (19 de agosto de 1990). Decreto Ley 1900 de 1990. Por el cual se reforman las normas y estatutos que regulan la actividad y servicios de telecomunicaciones y afines. Recuperado de http://www.mintic.gov.co/index.php/docs-no rmatividad? pid $=58 \&$ sid $=627: 1900$

--. (16 de julio de 2007). Ley 1150 de 2007. Por medio de la cual se introducen medidas para la eficiencia y la transparencia en la Ley 80 de 1993 y se dictan otras disposiciones generales sobre la contratación con Recursos Públicos. Recuperado de http://www. secretariasenado.gov.co/senado/basedoc/ ley/2007/ley_1150_2007.html

--. (30 de julio de 2009). Ley 1341 de 2009. Por la cual se definen principios y conceptos sobre la sociedad de la información y la organización de las Tecnologías de la Información y las Comunicaciones -TIC-, se crea la Agencia Nacional de Espectro y se dictan otras disposiciones. Recuperado de http://www. secretariasenado.gov.co/senado/basedoc/ ley/2009/ley_1341_2009.html

Constituyente. (1991). Constitución Política de Colombia. Recuperado de http://web.presidencia.gov.co/constitucion/index.pdf

Convención de París. (1919). Convención para la Reglamentación de la Navegación aérea Internacional. Recuperado de http://www. doctorcalleja.com.ar/Archivos/paris $\% 20$ 1919.htm

Convenio de Chicago. (1944). Convenio Sobre Aviación Civil Internacional. Recuperado de http://www.sct.gob.mx/fileadmin/DireccionesGrales/DGAC/Marco\%20Jur\%EDdico\%20 y\%20Regulatorio\%20Normativo/Juridico/ Convenios/Convenio\%20de\%20Chicago.pdf 
Corte Constitucional. (13 de octubre de 1993). Sentencia C-457 de 1993 M. P. Eduardo Cifuentes Muñoz. Recuperado de http:// www.secretariasenado.gov.co/senado/ basedoc/c-457_1993.htm

---. (23 de marzo de 2004). Sentencia C-278 de 2004 M.P Marco Gerardo Monroy Cabra y Manuel José Cepeda Espinosa. Recuperado de ww.secretariasenado.gov.co/senado/basedoc/cc_sc_nf/2004/c-278_2004.html

Declaración de Bogotá. (1976). La soberanía de Ios Estados Ecuatorial durante los correspondientes segmentos de la órbita geoestacionaria. Recuperado de http://www.booki.cc/ orbitando-satelites/declaracion-de-bogota/

Ferrer, M. (1976). Derecho Espacial. Buenos aires: Editorial Plus Ultra.

Kopal, V. (2009). Tratado sobre los principios que deben regir las actividades de los Estados en la exploración y utilización del espacio ultraterrestre, incluso la Luna y otros cuerpos celestes. Naciones Unidas. Recuperado de http://legal.un.org/avl/pdf/ha/tos/tos_s.pdf

Newton, I. (1687). Principia: Mathematical Principles of Natural Philosophy. (vol. I).

--. (1729). Axioms or Laws of Motion. The Mathematical Principles of Natural Philosophy. (Vol. I). London, UK: Tertia Aucta \& Emendata.

Peña, S. 2013, “¿Cuáles son las hipótesis posibles en cuanto a la accesión a las posiciones orbitales teniendo en cuenta el principio de equidad y los países en desarrollo? Texto final del seminario del profesor Alfredo Rey Córdoba para la Universidad de los Andes 2013-10.

Pound, R. (1910). Law in Books vs. Law in action. American Law Review, 44, 12-36.

Ramírez del Valle, B. (1985). La órbita sincrónica geoestacionaria: tercera dimensión de la soberanía nacional. Centro de pensamiento del Caribe Colombiano. Cartagena, Colombia: Editorial Costa Norte.

República de Colombia. (1998). Algunos aspectos relativos a la utilización de la órbita de los satélites geoestacionarios (A/AC.105/ C.2/L.200 y Corr.1.). Informe de la Subcomisión de Asuntos Jurídicos sobre la labor realizada en su $37^{\circ}$ período de sesiones (23 a 31 de marzo de 1998). Recuperado de http://www.oosa.unvienna.org/pdf/reports/ ac105/AC105_698S.pdf

Soler, R. (1974). Los satélites artificiales. BarceIona, España: Salvat Editores.

Unión Internacional de Telecomunicaciones. (1965). Convenio Internacional de Telecomunicaciones, Montreux. Recuperado de http:// www.itu.int/dms_pub/itu-s/oth/02/01/ S020100001F4018PDFS.pdf

---. (1973). Convenio Internacional de Telecomunicaciones, Málaga-Torremolinos. Recuperado de http://www.itu.int/dms_pub/itu-s/ oth/02/01/S020100001F4018PDFS.pdf 
--. (1980). Conferencia Administrativa Mundial de Radiocomunicaciones. Actas finales de la Conferencia Administrativa Mundial de Radiocomunicaciones, Ginebra 1979. Recuperada de http://www.itu.int/dms_pub/itu-s/ oth/02/01/S02010000394002PDFS.pdf

--. (1982). Convenio Internacional de Telecomunicaciones. Protocolo Final. Nairobi. Recuperado de http://www.itu.int/dms_pub/itu-s/ oth/02/09/s020900000b5201pdfs.pdf
--. (2011). Conjunto de textos fundamentales de la Unión Internacional de Telecomunicaciones adoptados por la Conferencia de Plenipotenciarios. Unión Internacional de Telecomunicaciones UIT. Recuperado de http:// www.itu.int/pub/S-CONF-PLEN-2011/es

--. (2012). Reglamento de radiocomunicaciones. Recuperado de http://www.itu.int/pub/ R-REG-RR-2012/es 